\title{
Avaliação ultrassonográfica de linfonodos na pesquisa de metástases de neoplasia mamária em cadelas ${ }^{1}$
}

\author{
Caterina Muramoto ${ }^{2}$, Franklin A. Sterman ${ }^{3}$, Stefano C.F. Hagen ${ }^{3}$, Ana Carolina B.C. Fonseca Pinto ${ }^{3}$, \\ Clair M. Oliveira ${ }^{3}$, Marcelo Faustino ${ }^{3}$, Mariana S.F. Talib3 e Luciana N. Torres ${ }^{4}$
}

\begin{abstract}
Muramoto C., Sterman F.A., Hagen S.C.F., Fonseca-Pinto A.C.B.C., Oliveira C.M., Faustino M., Talib M.S.F. \& Torres L.N. 2011. [Ultrasonographic evaluation of lymph nodes for metastasis research of canine mammary tumor.] Avaliação ultrassonográfica de linfonodos na pesquisa de metástases de neoplasia mamária em cadelas. Pesquisa Veterinária Brasileira 31(11):1006-1013. Setor de Diagnóstico por Imagem, Faculdade de Medicina Veterinária, Instituto de Saúde e Produção Animal, Universidade Federal Rural da Amazônia, Av. Presidente Tancredo Neves 2501, Belém, PA 66077-530, Brazil. E-mail: cmuramoto@gmail.com

Evaluation of lymph nodes is part of the staging of the mammary tumors and helps to establish a prognosis and therapy. B-mode ultrasonography was used to evaluate size, edge, nodal borders, shape, architecture, echotexture and echogenicity of lymph nodes and Doppler ultrasonography to evaluate the quantity and distribution of the vessels. The aims of this work were to identify which ultrasound features can be used to classify a lymph node as metastatic or non-metastatic; to establish its accuracy as a useful tool to this differentiation, and to establish procedures that permit the reproducibility of the results. Sixty seven lymph nodes of 30 female dogs with mammary tumors were examined by ultrasound and classified as metastatic or non-metastatic. The ultrasonographic diagnostic impression was confronted with the histopathologic results. The ultrasonographic classification of the lymph nodes was correct in $92.5 \%$ of the cases, with sensibility of $94.1 \%$, specificity of $92 \%$, positive predictive value of 0.8 , and negative predictive value of 0.9787 . The characteristics of the nodes to classify them as metastatic or non metastatic were registered. Ultrasound imaging of regional lymph nodes is important to detect metastases and it is suggested that it becomes a regular procedure in the staging of canine mammary tumors.
\end{abstract}

INDEX TERMS: Ultrasonography lymph nodes, mammary tumors, metastases, bitches.

RESUMO.- A avaliação de linfonodo, parte importante do estadiamento das neoplasias mamárias em cadelas, pode auxiliar no estabelecimento do prognóstico e na escolha da

\footnotetext{
${ }^{1}$ Recebido em 21 de abril de 2011.

Aceito para publicação em 29 de agosto de 2011.

${ }^{2}$ Setor de Diagnóstico por Imagem, Faculdade de Medicina Veterinária, Instituto de Saúde e Produção Animal (ISPA), Universidade Federal Rural da Amazônia (UFRA), Avenida Presidente Tancredo Neves 2501, CaixaPostal 917, Belém, PA 66077-530, Brasil. *Autor para correspondência: cmuramoto@gmail.com

${ }^{3}$ Departamento de Clínica Cirúrgica Veterinária, Faculdade de Medicina Veterinária e Zootecnia (FMVZ), Universidade de São Paulo (USP), Cidade Universitárias, Av. Prof. Orlando Marques de Paiva 87, São Paulo, SP 05508-270, Brasil.

${ }^{4}$ Departamento de Patologia, FMVZ-USP, Cidade Universitárias, São Paulo, SP 05508-270.
}

conduta terapêutica. A ultrassonografia em modo B possibilita avaliação de tamanho, contorno, borda, forma, arquitetura, ecotextura e ecogenicidade do parênquima dos linfonodos e, em modo Doppler, da quantidade e distribuição dos seus vasos internos. Este trabalho visou identificar as características ultrassonográficas mais importantes utilizadas para classificar os linfonodos em metastáticos e não-metastáticos, estabelecer elementos de confiabilidade do ultrassom como ferramenta para diferenciar linfonodos metastáticos de não-metastáticos e estabelecer procedimentos de reprodução deste exame. Foram examinados 67 linfonodos inguinais superficiais de 30 cadelas com tumor mamário e cada linfonodo foi classificado como metastático ou não-metastático. A impressão diagnóstica ultrassonográfica foi associada aos resultados do exame histopato- 
lógico dos linfonodos obtendo-se taxa de concordância de $92,5 \%$, índice de sensibilidade de $94,1 \%$, índice de especificidade de $92 \%$, valor preditivo positivo de 0,8 e valor preditivo negativo de 0,9787. Características ultrassonográficas que classificaram um linfonodo como metastático ou como não-metastático foram listadas. 0 exame ultrassonográfico dos linfonodos regionais constitui importante ferramenta na detecção de metástase e sugere-se incluí-lo como rotina do estadiamento de neoplasias mamárias em cadelas.

TERMOS DE INDEXAÇÃO: Ultrassonografia, linfonodos, neoplasias mamárias, metástases, cadelas.

\section{INTRODUÇÃO}

A neoplasia mamária é o tumor mais comum em cadelas não castradas (Sautet et al. 1992, Sorenmo 2003, Karayannopoulou et al. 2005) e o grau de comprometimento dos linfonodos regionais é fator prognóstico determinante para os pacientes que apresentam a forma maligna da doença (Hellmén et al. 1993, Sorenmo 2003, Karayannopoulou et al. 2005).

A disseminação dos tumores mamários costuma ocorrer por invasão local, crescimento intraductal mamário e pelo sistema linfático para posteriormente atingir a corrente sanguínea produzindo metástases à distância (Stavros 2006).

As glândulas mamárias abdominais caudais e inguinais são as mais frequentemente acometidas por neoplasias em cadelas (Sorenmo 2003). A linfa dessas mamas é drenada para o linfonodo inguinal superficial ipsilateral (Adams 1986, Sautet et al. 1992, Evans \& Delahunta 2001, Sorenmo 2003, Patsikas et al. 2006).

Várias técnicas de exame permitem a avaliação de linfonodos, cada uma com suas vantagens e limitações (Bruneton et al. 1998, Nyman et al. 2004) na tentativa de detectar alterações que contribuam para a diferenciação de características benignas e malignas de linfonodos (Nyman et al. 2004). A ultrassonografia constitui uma técnica segura, disponível (Torabi et al. 2004), de custo relativamente baixo e não invasiva. Os modos B e Doppler, quando associados, aumentam a sensibilidade e a especificidade da técnica na diferenciação dos processos de caráter benigno e maligno, mas a confirmação diagnóstica, de qualquer forma, somente será obtida pela avaliação citológica ou histopatológica (Blevins 2002, Dragoni et al. 1999, Eksioglu et al. 2003, Nyman et al. 2004).

Neste trabalho foi utilizada a técnica ultrassonográfica, em modo B e em modo Doppler de amplitude, para avaliação de linfonodos inguinais superficiais em cadelas com tumor na mama, visando complementar o estadiamento dessas pacientes e contribuir para o estabelecimento de ferramenta diagnóstica que preceda técnicas invasivas ou menos disponíveis.

Os objetivos deste trabalho foram: identificar as características ultrassonográficas mais importantes utilizadas para classificar os linfonodos inguinais superficiais em metastáticos e não-metastáticos; estabelecer elementos de confiabilidade do ultrassom como ferramenta para diferenciar linfonodos metastáticos de não-metastáticos e estabelecer procedimentos que permitam ao exame ultrassonográfico de linfonodos, para essa classificação, ser reprodutível.

\section{MATERIAL E MÉTODOS}

Participaram deste estudo 30 cadelas com tumor em pelo menos uma das mamas inguinais ou abdominais caudais, atendidas no Serviço de Obstetrícia e Ginecologia do Hospital Veterinário da Faculdade de Medicina Veterinária e Zootecnia, Universidade de São Paulo (Hovet-FMVZ-USP). Foram avaliados 67 linfonodos inguinais superficiais (um ou mais por cão), por um examinador único e cego para o resultado final, por meio de exame ultrassonográfico em modo B (ou modo convencional que adquire uma imagem bidimensional em tons de cinza) e em modo Doppler de amplitude (ou power Doppler, mais sensível que o Doppler colorido na detecção de sinais mais fracos provenientes de vasos sanguíneos menores). Um estudo de dimensionamento amostral realizado, com dados parciais, pelo Centro de Estatística Aplicada do Instituto de Matemática e Estatística da Universidade de São Paulo sugeriu avaliação de um número mínimo de 51 linfonodos para se obter confiança de $95 \%$ e um erro máximo de $6 \%$.

Utilizou-se o aparelho de ultrassom ATL $^{5}$ modelo HDI 5000, munido de transdutor linear multifrequencial de 5-12 MHz e programa computacional pré-ajustado para pequenas partes/superficial.

Para localização do linfonodo inguinal superficial, o cão é colocado em decúbito dorsal e o transdutor é posicionado em sentido longitudinal e paralelo à mama inguinal, sem excesso de pressão contra a pele e, então, realizam-se movimentos de varredura oblíquos e laterais, mantendo o transdutor em sentido longitudinal, até a localização do linfonodo. Localizado o linfonodo, são realizados os cortes de varredura longitudinais e transversais do órgão, verificando suas características ultrassonográficas. Não foi utilizada sedação nos cães deste estudo para a realização do exame ultrassonográfico.

Pelo exame ultrassonográfico em modo B foram avaliadas as características tamanho (comprimento, espessura, largura e volume), contorno (regular ou irregular), borda (definida ou pouco definida), forma (ovalada, arredondada, amorfa), arquitetura (preservada ou desorganizada), ecotextura (preservada, homogênea, heterogênea ou grosseira) e ecogenicidade (hipoecogênica, hiperecogênica, mista ou normal).

Com relação à forma, foi calculada a razão entre comprimento e espessura, denominada razão $\mathrm{C} / \mathrm{E}$. Os linfonodos com valores de razão $\mathrm{C} / \mathrm{E}$ maior que 2 tenderam a manter a forma ovalada/ alongada e os com valor menor que 2 apresentaram tendência à forma mais arredondada/globosa.

No modo Doppler de amplitude, foram avaliadas características relacionadas à vascularização no que se refere à quantidade (discreta/pequena ou moderada/elevada) e localização (hilar, periférica ou mista).

Após o exame ultrassonográfico, o linfonodo foi classificado em metastático ou não-metastático, conforme avaliação do examinador e o cão, encaminhado para o procedimento cirúrgico. Os procedimentos cirúrgicos foram preconizados de acordo com a

\footnotetext{
${ }^{5}$ Philips Medical System ${ }^{\circledR}$, Philips do Brasil Ltda, Av. Dr. Marcos Penteado de Ulhôa Rodrigues 939, Torre Jacarandá, Tamboré, Barueri, São Paulo.
} 
rotina do Serviço de Obstetrícia e Ginecologia do Hovet-USP e tanto o tecido mamário como o linfonodo inguinal superficial eram encaminhados para estudo histopatológico (padrão-ouro para o diagnóstico), conduzido segundo as normas do Serviço de Patologia Animal da FMVZ-USP (classificação de tumores mamários de cães sugerida pela Organização Mundial de Saúde, descrita por Misdorp et al. 1999). Os cães que apresentavam tumor em mais de uma mama, de tipos histológicos distintos, considerou-se para o estudo o diagnóstico histológico de pior grau.

Para a avaliação estatística da associação entre cada característica analisada no exame ultrassonográfico e o diagnóstico histopatológico do linfonodo em metastático ou não-metastático foram utilizados os testes: Teste Qui-quadrado de Pearson $\left(\mathrm{X}^{2}\right)$, Teste exato de Fisher (Fisher), Teste de Wilcoxon e Teste t-Student.

0 nível de significância estabelecido para análise foi de $5 \%$ $(\mathrm{p}<0,05)$, o que implica que o erro máximo permitido ao afirmar que existe associação entre determinada característica e o tipo de linfonodo é de $5 \%(p=0,05)$. Quanto menor o valor de $p$, menor a probabilidade de erro.

\section{RESULTADOS}

Os tumores do tipo maligno foram identificados em 21 (70\%) cadelas e os do tipo benigno em nove (30\%). Das 21 cadelas que apresentaram a forma maligna do tumor, sete $(33,3 \%)$ apresentaram comprometimento metastático dos linfonodos inguinais superficiais. $\mathrm{Na}$ avaliação histopatológica dos linfonodos, 50 (74,6\%) deles foram classificados como não-metastáticos e 17 (25,4\%) como metastáticos.

Na avaliação ultrassonográfica, 47 (70,1\%) linfonodos foram classificados como não-metastáticos (sendo 46 concordantes com o exame histopatológico) e 20 (29,9\%) classificados como metastáticos (sendo 16 concordantes com o exame histopatológico).
0 índice de sensibilidade foi de 94,1\% (16/17), o de especificidade foi de $92 \%$ (46/50) e a precisão diagnóstica, ou seja, a proporção de acertos do exame ultrassonográfico foi de $92,5 \%$ (62/67). 0 valor preditivo positivo (VP+) foi de $0,8(16 / 20)$ e o valor preditivo negativo (VP-) foi de 0,9787 (46/47).

No cão, o linfonodo inguinal superficial normal, ao exame ultrassonográfico, é um órgão achatado, com forma elíptica, em que é possível identificar um córtex relativamente hipoecogênico e hilo e mediastino relativamente hiperecogênicos com ecotextura um pouco mais grosseira que a cortical. Ao modo Doppler, os vasos sanguíneos são identificados apenas na região hilar.

0 aparelho de alta resolução empregado neste estudo, utilizando transdutor multifrequencial de 5 a $12 \mathrm{MHz}$, pré-ajustado para pequenas partes/superficial, foi adequado para a localização dos linfonodos inguinais superficiais de todas as cadelas e para a identificação das características ultrassonográficas pesquisadas.

0 comprimento, espessura e largura dos linfonodos não-metastáticos variaram de, respectivamente, 0,42 a $2,69 \mathrm{~cm}$ (média, $\bar{x} \bar{x}$ de $1,48 \mathrm{~cm}$ e desvio padrão, $\sigma=$ $0,61 \mathrm{~cm}), 0,15$ a $0,64 \mathrm{~cm}(\bar{x} \bar{x}=0,38 \mathrm{~cm}$ e $\sigma=0,16 \mathrm{~cm})$ e 0,28 a $1,34 \mathrm{~cm}(\bar{x} \bar{x}=0,67 \mathrm{~cm}$ e $\sigma=0,24 \mathrm{~cm})$ e os valores para os linfonodos metastáticos variaram de, respectivamente, 0,85 a $3,98 \mathrm{~cm}(\bar{x} \bar{x}=2,25 \mathrm{~cm}$ e $\sigma=0,89), 0,58$ a $2,26 \mathrm{~cm}$ $(\bar{x} \bar{x}=1,06 \mathrm{~cm}$ e $\sigma=0,4 \mathrm{~cm})$ e 0,55 a $2,68(\bar{x} \bar{x}=1,45 \mathrm{~cm}$ e $\sigma=0,53 \mathrm{~cm})$.

0 teste $t$-Student mostrou haver evidências de diferença $(\mathrm{p}<0,0033$ para comprimento e $\mathrm{p}<0,001$ para espessura e largura) entre os dois grupos, metastático e não-metastático, havendo uma tendência dos metastáticos apresentarem comprimento, largura e espessura significativamente maiores que o grupo não-metastático.

Quadro 1. Frequência (em número e porcentagem) de cada característica ultrassonográfica avaliada nos modos B e Doppler de amplitude nos linfonodos não-metastáticos e metastáticos, com os respectivos níveis descritivos (p) e o teste estatístico aplicado

\begin{tabular}{|c|c|c|c|c|c|c|}
\hline \multirow[t]{2}{*}{ Característica } & \multirow[t]{2}{*}{ Classificação } & \multicolumn{2}{|c|}{$\begin{array}{c}\text { Não-metastático } \\
n=50\end{array}$} & \multicolumn{2}{|c|}{$\begin{array}{c}\text { Metastáticos } \\
n=17\end{array}$} & \multirow[t]{2}{*}{$\mathrm{P}$} \\
\hline & & $\mathrm{n}$ & $\%$ & $\mathrm{n}$ & $\%$ & \\
\hline \multirow[t]{2}{*}{ Contorno } & Regular & 44 & 88 & 7 & 41,2 & $<0,001$ \\
\hline & Irregular & 6 & 12 & 10 & 58,8 & Fisher \\
\hline \multirow[t]{2}{*}{ Borda } & Definida & 41 & 82 & 17 & 100 & $<0,098$ \\
\hline & Pouco definida & 9 & 18 & 0 & 0,0 & Fisher \\
\hline \multirow[t]{3}{*}{ Forma } & Ovalada & 49 & 98 & 8 & 47,1 & $<0,001$ \\
\hline & Arredondada & 0 & 0,0 & 6 & 35,3 & Fisher \\
\hline & Amorfa & 1 & 2 & 3 & 17,6 & \\
\hline \multirow[t]{2}{*}{ Razão C/E } & Maior que 2 & 50 & 100 & 10 & 58,8 & $<0,001$ \\
\hline & Menor que 2 & 0 & 0,0 & 7 & 41,2 & Fisher \\
\hline \multirow[t]{2}{*}{ Arquitetura } & Preservada & 44 & 88 & 3 & 17,6 & $<0,001$ \\
\hline & Desorganizada & 6 & 12 & 14 & 82,4 & $X^{2}$ \\
\hline \multirow[t]{4}{*}{ Ecotextura } & Preservada & 34 & 68 & 1 & 5,8 & $<0,001$ \\
\hline & Grosseira & 12 & 24 & 6 & 35,3 & Fisher \\
\hline & Homogênea & 4 & 8 & 6 & 35,3 & \\
\hline & Heterogênea & 0 & 0,0 & 4 & 23,5 & \\
\hline \multirow[t]{3}{*}{ Ecogenicidade } & Normal & 42 & 84 & 1 & 5,9 & $<0,001$ \\
\hline & Hipoecogênica & 6 & 12 & 12 & 70,6 & Fisher \\
\hline & Hiperecogênica & 2 & 4 & 4 & 23,5 & \\
\hline \multirow[t]{2}{*}{ Quantidade de vascularização } & Discreta/pequena & 38 & 76 & 5 & 29,4 & $<0,0005$ \\
\hline & Moderada/elevada & 12 & 24 & 12 & 70,6 & $\mathrm{X}^{2}$ \\
\hline \multirow[t]{3}{*}{ Localização da vascularização } & Hilar & 34 & 68 & 0 & 0,0 & $<0,001$ \\
\hline & Mista & 16 & 32 & 8 & 47,1 & $\mathrm{X}^{2}$ \\
\hline & Periférica & 0 & 0,0 & 9 & 52,9 & \\
\hline
\end{tabular}



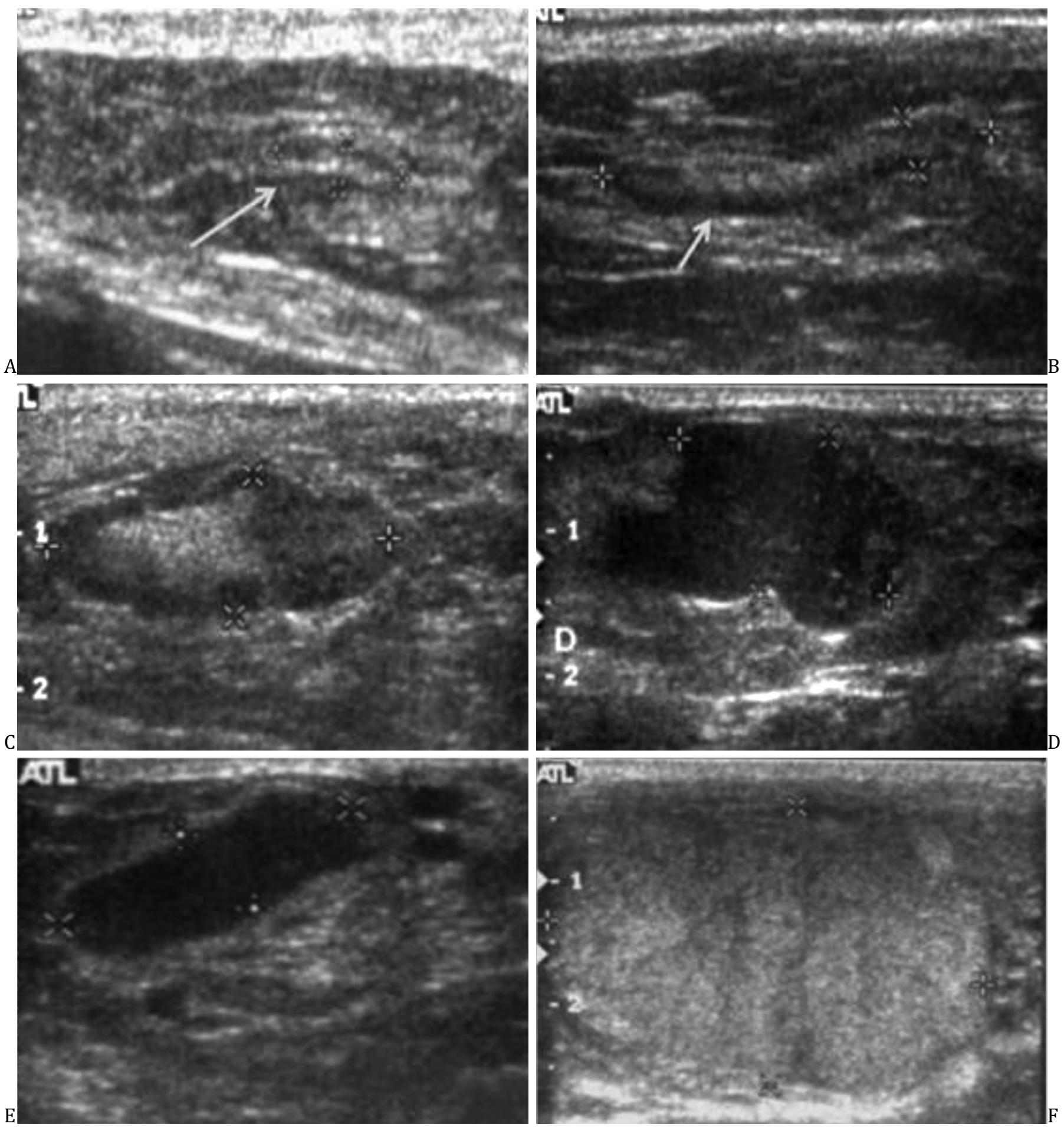

Fig.1. Imagens ultrassonográficas de linfonodos inguinais superficiais de cadelas com tumor na mama. 0 diagnóstico histopatológico do linfonodo e do tumor (Dx) e as características ultrassonográficas em modo B estão apresentadas para cada imagem identificada de A até F. (A) Linfonodo não-metastático (Dx: carcinoma simples túbulo-papilífero grau II); contorno regular, borda pouco definida, ovalado, arquitetura e ecotextura preservadas, ecogenicidade normal, razão C/E:3,88. (B) Linfonodo não-metastático (Dx: carcinoma papilífero simples); contorno regular, borda definida, ovalado/alongado, arquitetura e ecotextura preservadas, ecogenicidade normal, razão C/E:8,06. (C) Linfonodo não-metastático (Dx: carcinoma complexo grau I); contorno irregular, borda definida, ovalado, arquitetura preservada, ecotextura grosseira, ecogenicidade normal, razão C/E:2,68. (D) Linfonodo metastático (Dx: carcinoma simples sólido invasivo grau III); contorno irregular, borda definida, amorfo, arquitetura desorganizada, ecotextura grosseira, hipoecogênico, razão C/E:1,58. (E) Linfonodo metastático (Dx: carcinoma simples sólido invasivo grau III); contorno regular, definido, ovalado, arquitetura desorganizada, ecotextura homogênea, hipoecogênico, razão C/E: 2,78. (F) Linfonodo metastático (Dx: carcinoma simples anaplásico grau III); contorno irregular, borda definida, arredondado, arquitetura desorganizada, ecotextura heterogênea, hiperecogênico, razão C/E: 1,46. 
Quanto ao volume, em função dos elevados valores de desvio padrão, considerou-se que as médias não eram boas medidas de tendência central e então se utilizou o Teste de Wilcoxon que compara as medianas. A mediana do grupo não-metastáticos foi de $0,17 \mathrm{~cm}^{3}$ e a dos metastáticos foi de $1,52 \mathrm{~cm}^{3}$. 0 Teste de Wilcoxon evidenciou que o grupo dos metastáticos apresentou volume mediano significativamente maior do que o grupo dos não-metastáticos, considerando $\mathrm{p}<0,001$.

As quantidades e porcentagens dos 50 linfonodos inguinais superficiais não-metastáticos e dos 17 linfonodos metastáticos com determinada característica ultras-sono-
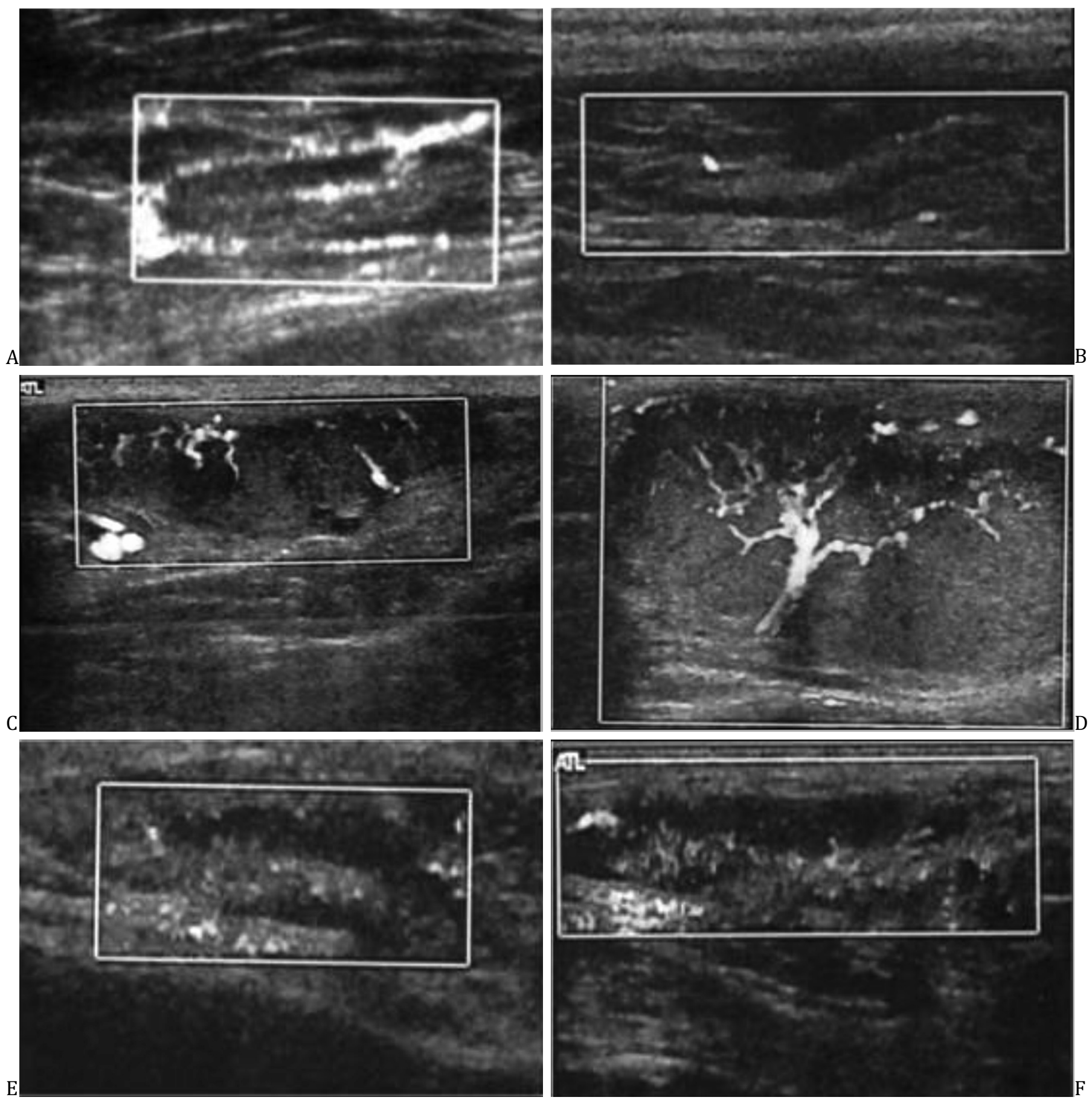

Fig.2. Imagens ultrassonográficas de linfonodos inguinais superficiais de cadelas com tumor na mama. 0 diagnóstico histopatológico do linfonodo e do tumor (Dx) e as características ultrassonográficas em modo Doppler de amplitude estão apresentadas para cada imagem identificada de $A$ até F. (A) Linfonodo não-metastático (Dx: carcinoma simples túbulo-papilífero grau I); vascularizaçaão moderada e hilar. (B) Linfonodo não-metastático (Dx: carcinoma papilífero simples); vascularização discreta/pequena e hilar. (C) Linfonodo metastático (Dx: carcinoma simples anaplásico grau III); vascularização moderada e periférica. (D) Linfonodo metastático (Dx: carcinoma simples anaplásico grau III); vascularização elevada e mista. (E) Linfonodo metastático (Dx: carcinoma simples papilífero grau I); vascularização moderada e mista. (F) Linfonodo não-metastático (Dx: carcinoma complexo grau I); vascularização moderada/elevada e mista. 
gráfica e o respectivo nível descritivo (p) para cada item avaliado estão dispostos no Quadro 1.

\section{DISCUSSÃO}

A busca por métodos menos invasivos que avaliem o linfonodo no momento pré-operatório tem obtido resultados animadores com o uso do ultrassom como ferramenta para diferenciar processos benignos e malignos dos linfonodos (Esen et al. 2005). Diferentes padrões de resposta a estímulos antigênicos no linfonodo podem ser gerados e a identificação dessas respostas pode colaborar para diferenciar uma linfadenomegalia de aspecto maligno de uma benigna.

Apesar da evidente tendência dos linfonodos metastáticos apresentarem tamanho maior que os não-metastáticos, a grande variabilidade dentro do mesmo grupo de linfonodos (desvios-padrão elevados em ambos os grupos), impediu o estabelecimento de um valor padrão que os classificasse como de um ou de outro tipo. Variações no tamanho sofrem influência do tempo decorrido desde a instalação do quadro inflamatório ou metastático, da resposta imune do linfonodo frente ao processo instalado e também, da variabilidade racial da espécie canina, com falta de dados na literatura sobre padronização de tamanho de linfonodos por porte em cães.

À semelhança do trabalho de Nyman et al. (2005), encontrou-se tendência do linfonodo não-metastático apresentar contorno regular e do metastático, irregular. Irregularidades no contorno podem ser justificadas pela proliferação de células tumorais nos seios subcapsulares, o que pode produzir abaulamentos focais ou lobulações no contorno externo (Nyman et al. 2005, Stavros 2006).

Nyman et al. (2005) observaram tendência a melhor definição das bordas tanto nos linfonodos inflamatórios como nos com características malignas e atribuíram tal fato à maior diferença de impedância acústica entre o tecido alterado do linfonodo e o tecido adjacente. Diferentemente, nos linfonodos normais a ecogenicidade é, frequentemente, semelhante ao tecido adjacente, dificultando a diferenciação do contorno, o que pode justificar os $18 \%$ de linfonodos não-metastáticos que apresentaram bordas pouco definidas.

A forma do linfonodo mostrou associação significativa com o diagnóstico do linfonodo, semelhante ao observado em outros trabalhos (Vassalo et al. 1992, Esen et al. 2005 e Nyman et al. 2005). Verificou-se que todos os linfonodos não-metastáticos apresentaram razão C/E maior que dois, indicando manutenção da forma oval, o que pode ser explicado pela tendência ao aumento proporcional em todos os planos do linfonodo na linfadenomegalia de origem inflamatória, de acordo com Vassalo et al. (1992), Bruneton et al. (1998), Chammas et al. (2004) e Stavros (2006). Contrariamente, no caso dos linfonodos metastáticos, um pouco mais da metade teve alteração na forma normal e $41,2 \%$ dos 17 linfonodos apresentaram razão C/E menor que dois, indicando tendência à forma arredondada, explicada pelo crescimento desproporcional do eixo menor do linfonodo, nos comprometimentos neoplásicos, de acordo com Vassalo et al. (1992), Bruneton et al. (1998), Chammas et al.
(2004) e Stavros (2006).

Houve diferença significativa da arquitetura entre os dois grupos, com tendência do linfonodo não-metastático em apresentar arquitetura preservada e do metastático em apresentar arquitetura desorganizada, sem identificação do hilo hiperecogênico central (Vassalo et al. 1992, Bruneton et al. 1998, Chammas et al. 2004, Salwei et al. 2005, Stavros 2006).

Como as metástases costumam atingir primeiramente os seios subcapsulares e corticais, causando comprometimento centrípeto progressivo, afilando ou empurrando o mediastino para uma das bordas, costuma-se associar a perda total da visualização do hilo hiperecogênico ao envolvimento metastático de grau avançado (Esen et al. 2005).

Vassalo et al. (1992) relatam que a perda da identificação do hilo hiperecogênico central pode ser decorrente de áreas de necrose central presentes tanto em processos metastáticos como em algumas condições inflamatórias mais graves ou crônicas e Salwei et al. (2005) citam ainda a sua ocorrência por infiltração de células tumorais e por hiperplasia linfoide secundária a processo inflamatório ou infeccioso com desenvolvimento de novos centros germinativos no interior do hilo.

Apesar de ter sido verificada tendência dos linfonodos metastáticos apresentarem ecotextura homogênea ou heterogênea e dos não-metastáticos apresentarem-na preservada, o fato de serem encontradas as categorias de classificação preservada, grosseira ou homogênea em ambos os grupos indica que essa característica colabora na diferenciação, mas não deve ser usada como critério único. Considerando-se que para a determinação das categorias homogênea ou heterogênea há concomitantemente perda da arquitetura, sugere-se que ambas as características, ecotextura e arquitetura, sejam avaliadas em conjunto.

Acredita-se que a ecotextura heterogênea possa ocorrer pela existência de áreas de necrose de liquefação ou hemorrágica, ou áreas que entremeiam tecido normal e tecido com alterações tumorais (Bruneton et al. 1998, Chammas et al. 2004, Nyman et al. 2005, Kinns \& Mai 2007), ou ainda, pela presença de microcalcificações e calcificações grosseiras nos linfonodos (Bruneton et al. 1998, Chammas et al. 2004).

Foram encontrados $94,1 \%$ dos linfonodos metastáticos com alteração na ecogenicidade, comumente com redução do tom de cinza normal. Porém, assim como descrito por Stavros (2006), a redução da ecogenicidade por elevação da celularidade pode ocorrer também na hiperplasia linfoide.

Apesar da maior probabilidade do linfonodo não-metastático apresentar vascularização discreta ou pequena e do linfonodo metastático apresentar vascularização moderada ou elevada, observaram-se exceções em ambos os casos. No grupo de linfonodos não-metastáticos, a detecção de fluxo moderado ou elevado provavelmente está associada aos linfonodos de caráter inflamatório em cujo processo há aumento do fluxo e do diâmetro vascular (Chammas et al. 2004, Nyman et al. 2005). No grupo de linfonodos metastáticos com vascularização discreta ou pequena, acredita-se que focos de isquemia, necrose ou doença tromboem- 
bólica possam causar áreas hipoperfundidas ou até mesmo aspecto avascular (Salwei et al. 2005).

A localização da vascularização no interior do linfonodo apresentou forte associação com o diagnóstico, havendo tendência da vascularização ser hilar nos não-metastáticos e de ser do tipo periférica ou mista no grupo dos metastáticos, notando-se que em nenhum linfonodo não-metastático o fluxo foi do tipo periférico e em nenhum metastático a vascularização foi do tipo hilar, em concordância com os trabalhos de Tschammler et al. (1996), Eksioglu et al. (2003), Esen et al. (2005) e Nyman et al. (2005).

Uma das hipóteses para o deslocamento dos vasos hilares levantadas por Salwei et al. (2005) e Steinkamp et al. (2002) é a de que proliferação de células neoplásicas possa gerar um efeito de massa e alterar o curso normal dos vasos hilares, deslocando-os para a periferia; quanto aos vasos aberrantes, existe a possibilidade de representarem neovascularização resultante da angiogênese. Steinkamp et al. (2002) relatam que o tipo de padrão vascular com aspecto pontilhado, periférico ou misto, depende do tempo de evolução do processo metastático, do grau de infiltração celular e de angiogênese e da presença de áreas de necrose.

Os linfonodos avaliados neste estudo apresentaram, em muitos casos, vasos com calibre muito pequeno, impossibilitando a análise espectral, a qual inclui o cálculo dos valores de IP (índice de pulsatilidade) e IR (índice de resistividade), pois o menor volume amostral fornecido pelo aparelho ultrapassava o diâmetro do vaso sanguíneo. 0 volume de amostra inadequado (como no caso de ultrapassar o diâmetro do vaso) leva a resultados incoerentes, por exemplo, por captação de movimentos das paredes dos vasos ou por registro simultâneo de espectros venoso e arterial em um mesmo traçado (Cerri et al. 1996). 0 valor diagnóstico das medidas desses índices tem sido contraditório nos diferentes trabalhos em que foram avaliados.

As Figuras 1 e 2 contêm alguns exemplos de imagens ultrassonográficas de linfonodos inguinais superficiais deste estudo, respectivamente, em modo B e em modo Doppler de amplitude.

Os resultados deste trabalho indicam não haver característica ultrassonográfica absoluta para categorizar o linfonodo como metastático ou não-metastático, mas há maior ou menor tendência de associação de determinadas características com o diagnóstico, assim como discutido por Chammas et al. (2004).

Num país como o Brasil, em que há de se considerar as dificuldades financeiras de grande parcela da população e em que ainda não há disponibilidade na rotina da medicina veterinária de técnicas cintilográficas e de modalidades anátomo-funcionais, o ultrassom se mostra como um exame não invasivo, que traz informações importantes na diferenciação de linfonodos com e sem comprometimento metastático e que apresenta custo relativamente acessível, além de ser técnica disponível, principalmente em grandes centros urbanos.

\section{CONCLUSÕES}

Deve-se considerar grande a possibilidade de comprometimento metastático, na presença de linfonodo com o conjunto de características: contorno irregular, forma arredondada com razão C/E menor que 2, arquitetura desorganizada e com ecotextura homogênea ou heterogênea, ecogenicidade alterada, vascularização elevada e do tipo periférica, em função da forte associação entre tais características e o diagnóstico metastático.

Para o grupo dos linfonodos não-metastáticos, sugere-se que na presença das características ultrassonográficas: contorno regular, forma ovalada com razão C/E maior que 2 , arquitetura e ecotextura preservadas, ecogenicidade normal, vascularização discreta a pequena e de localização hilar, considere-se maior a possibilidade de estar diante de um linfonodo não-metastático.

Os altos índices de sensibilidade $(94,1 \%)$ e de especificidade $(92 \%)$ do exame ultrassonográfico na diferenciação de linfonodos metastáticos de não-metastáticos em cadelas com neoplasia mamária obtidos neste trabalho, somados à elevada taxa de concordância diagnóstica (92,5\%) entre os exames ultrassonográfico e histopatológico e aos elevados valores de VP+ $(0,8)$ e VP- $(0,9787)$, indicam que, para esta avaliação, o ultrassom foi uma técnica bem sucedida e confiável.

Sugere-se que, quando o exame ultrassonográfico em cadelas com neoplasia mamária indicar a presença de metástase no linfonodo, se prossiga a investigação para certificar-se do diagnóstico, pois, apesar de existir uma chance muito grande do linfonodo realmente ser metastático, há que se considerar $20 \%$ de falso-positivos (VP+ igual a 0,8). De outra forma, frente a uma sugestão diagnóstica de processo não-metastático existe uma chance mínima de não o ser, pois os falso-negativos foram de apenas $2,2 \%$.

0 exame ultrassonográfico dos linfonodos regionais constitui importante ferramenta com alta confiabilidade na detecção de metástase e sugere-se incluí-lo como rotina do estadiamento de neoplasias mamárias em cadelas.

\section{REFERÊNCIAS}

Adams D.R. 1986. Canine Anatomy: a systemic study. The Iowa State University Press, Ames, p.379-397.

Blevins W.E. 2002. Ultrasonography for cancer diagnosis and monitoring, p.565-572. In: Morrison W.B. (Ed.), Cancer in Dogs and Cats: Medical and surgical management. Teton New Media, Jackson.

Bruneton J.N., Rubatelli L. \& Solbiati L. 1998. Linfonodos, p.279-301. In: Solbiati L. \& Rizzatto G. (Eds), Ultra-sonografia das Estruturas Superficiais: Alta frequência, Doppler e procedimentos intervencionistas. Revinter, Rio de Janeiro.

Cerri G.G., Mólnar L.J. \& Vezozzo D.C.P. 1996. Doppler. Sarvier, São Paulo, p.15-30.

Chammas M.C., Saito O.C. \& Cerri G.G. 2004. Linfonodos cervicais, p.155170. In: Saito O. (Ed.), Ultra-sonografia de Pequenas Partes. Revinter, Rio de Janeiro.

Dragoni F., Cartoni C., Pescarmona E., Chiarotti F., Puopolo M., Orsi E., Pignoloni P., De Gregoris C. \& Mandelli F. 1999. The role of high resolution pulsed and color Doppler ultrasound in the differential diagnosis of benign and malignant lymphadenopathy. Cancer 85:2485-2490.

Ekşioğlu A.S., Özdemir A. \& Özdemir H. 2003. Aksiller lenfadenopatilerin ayırıcı tanısında gri-skala ve renkli-power Doppler ultrasonografi. Tanı Girişim Radyol. 9(4):445-451. 
Esen G., Gurges B., Yilmaz M.H., Ilvam S., Ulus S., Celik V., Farahmand M. \& Calay 0.0. 2005. Gray scale and power Doppler US in the preoperative evaluation of axillary metastases in breast cancer patients with no palpable lymph nodes. Eur. Radiology 15:1215-1223.

Evans H.E. \& De Lahunta A. 2001. Guia para a Dissecção do Cão. Guanabara Koogan, Rio de Janeiro.

Hellmén E., Bergström R., Holmberg L., Spắngberg I.B., Hansson K. \& Lindgren A. 1993. Prognostic factors in canine mammary tumours: A multivariate study of 202 consecutive cases. Vet. Pathol. 30:20-27.

Karayannopoulou M., Kaldrymidou E., Constantinidis T.C. \& Dessiris A. 2005. Histological grading and prognosis in dogs with mammary carcinomas: Application of a human grading method. J. Comp. Pathol. 133:246-252.

Kinns J. \& Mai W. 2007. Association between malignancy and sonographic heterogeneity in canine and feline abdominal lymph nodes. Vet. Radiol. Ultrasound 48:565-569.

Misdorp W., Else R.W., Hellmen E. \& Lipscomb T.P. 1999. WHO Histological Classification of Mammary Tumors of the Dog and the Cat. Vol.7. Institute of Pathology of Armed Forces, Washington, DC.

Nyman H.T., Kristensen A.T., Flagstad A. \& McEvoy F.J. 2004. A review of the sonographic assessment of tumour metastases in liver and superficial lymph nodes. Vet. Radiol. Ultrasound 45:438-448.

Nyman H.T., Kristensen A.T., Skovgaard I.M. \& Mcevoy F.J. 2005. Characterization of normal and abnormal canine superficial lymph nodes using grayscale B-mode, color flow mapping, power, and superficial Doppler ultrasonography: A multivariate study. Vet. Radiol. Ultrasound 46:404-410.
Patsikas M.N., Karayannopoulou M., Kaldrymidoy E., Papazoglou L.G., Papadopoulou P.L. \& Tzegas S.I. 2006. The lymph drainage of the neoplastic mammary glands in the bitch: A lymphographic study. Anat. Histol. Embryol. 35:228-234.

Salwei R.M., OBrien R.T. \& Matheson J.S. 2005. Characterization of lymphomatous lymph nodes in dogs using contrast harmonic and power Doppler ultrasound. Vet. Radiol. Ultrasound 46:411-416.

Sautet J.Y., Ruberte J., Lopez C., Gine J.M., Ordoñez G. \& Cingia A. 1992. Lymphatic system of the mammary glands in the dog: An approach to the surgical treatment of malignant mammary tumours. Canine Pract., Surgery 17:30-33.

Sorenmo K. 2003. Canine mammary gland tumors. Vet. Clin. North Am., Small Anim. Pract. 33:573-596.

Stavros A.T. 2006. Ultra-sonografia da Mama. Guanabara Koogan, Rio de Janeiro, p.783-822.

Steinkamp H.J., Wissgott C., Rademaker J. \& Felix R. 2002. Current status of power Doppler and color Doppler sonography in the differential diagnosis of lymph node lesions. Eur. Radiology 12:1785-1793.

Torabi M., Aquino S.L. \& Harisinghani M.G. 2004. Current concepts in lymph node imaging. J. Nuclear Med. 45:1509-1518.

Tschammler A., Wirkner H., Ott G. \& Hahn D. 1996. Vascular patterns in reactive and malignant lymphadenopathy. Eur. Radiology 6:473-480

Vassalo P., Wernecke K., Roos N. \& Peters P.E. 1992. Differentiation of benign from malignant superficial lymphadenopathy: The role of high-resolution US. Radiology 183:215-220. 